\title{
Study of the surface state of nickel electrode during polarization in an alkaline medium by electrochemical impedance spectroscopy method
}

\author{
V.E. Kasatkin,*ib L.P. Kornienko, A.I. Shcherbakov, I.G. Korosteleva, \\ I.V. Kasatkina and V.N. Dorofeeva
}

A.N. Frumkin Institute of Physical Chemistry and Electrochemistry, Russian Academy of Sciences, Leninsky pr. 31, 119071 Moscow, Russian Federation

*E-mail: vadim_kasatkin@mail.ru

\begin{abstract}
The state of nickel electrode surface in a weakly alkaline medium was investigated by the Electrochemical Impedance Spectroscopy method (EIS). This system was chosen as a model where nickel has sufficient stability, which makes it possible to minimize the probability of a significant change in the electrode properties in the course of a single measurement of the impedance spectrum and to carry out studies in a wide range of potentials. The impedance spectra obtained under the conditions of potentiostatic and galvanostatic polarization turned out to be similar in both modes. An equivalent electrical circuit of the processes occurring in an oxidehydroxide film and at its boundaries with a metal and a solution is proposed, which adequately and with good accuracy describes these processes and allows to evaluate the properties the electrical circuit elements. The regularities of the experimental conditions influence on the parameters of the equivalent circuit elements are discussed. At potentials not exceeding $+400 \ldots+500 \mathrm{mV}$, two characteristic semicircles are observed on the Nyquist diagram. In the region of higher anodic polarization, the shape of the low-frequency semicircle is deformed, taking the form of a pseudo inductive loop, characteristic for the active dissolution of the electrode metal. It was found that the high-frequency region of the impedance spectrum is practically independent of the electrode potential and is associated with the presence of an oxide-hydroxide layer on the nickel surface. The possibility to distinguish the processes of Faraday oxidation of nickel at the metal-film interface and the ionic charge transfer at the film-solution interface is revealed. Capacitive and resistive parameters of the studied processes are calculated.
\end{abstract}

Keywords: nickel, alkaline, pitting corrosion, electrochemical impedance spectroscopy method.

Received: November 12, 2020. Published: December 7, 2020

doi: $\underline{10.17675 / 2305-6894-2020-9-4-21}$

\section{Introduction}

Nickel is widely used as a structural material in many fields of technology [1] due to its high passivability and corrosion resistance in an alkaline environment [2], in particular, in the electrolysis industry, in alkaline batteries, as electrode materials [3-5]. Nickel-based 
catalysts are actively used to intensify catalytic and electrocatalytic processes [6,7]. As an alloying element, nickel can, to a significant extent, transfer its unique resistance in alkali solutions to high-nickel steels and cast iron [8]. The most important characteristics of nickel are the capacity and conductivity of the surface layers, as well as resistance to general and pitting corrosion. Resistance to pitting corrosion is largely determined by the properties of the passivating film. In this regard, it is relevant to study the structure and properties of the passivating layer, its boundaries with the metal and solution, and the mechanism of chemical and electrochemical reactions at these boundaries.

The anodic behavior of nickel in an alkaline medium has been investigated by electrochemical and physicochemical methods [9-19]. Based on a combination of the results of many works, G. Shtreblov $[13,14]$ concluded that a passive film on nickel has a two-layer structure and consists of $\mathrm{NiO}$ (inner layer) and $\mathrm{Ni}(\mathrm{OH})_{2}$ (outer layer). In situ studies results on nickel pitting model using Raman spectroscopy and EIS [15] fully confirmed this conclusion. In [16], the formation of films on nickel, their thickness, and the chloride ion distribution were studied in situ by the radioisotope method and XPS. The ellipsometric measurements [16, 17] were carried out in combination with repeated cycling on polycrystalline nickel in $0.1 \mathrm{~N} \mathrm{KOH}$. It is shown in [16] that in the potential range of $+0.3 \ldots+1.35 \mathrm{~V}$, the phase composition does not change and corresponds to the $\mathrm{Ni}(\mathrm{OH})_{2}$ stoichiometric formula. When exposed in an open circuit state (at $E=+0.7 \ldots+0.9 \mathrm{~V}$ ) for 10-30 hours, apparently, an electrochemically inactive subsurface oxide $\mathrm{NiO}_{z}$ is formed. According to the data of [17], a poorly conducting oxide is formed on the reduced nickel surface, and at higher potentials, a highly conducting one. At the potentials above $+1 \mathrm{~V}$, nickel oxide has the composition $\mathrm{NiO} \times \mathrm{H}_{2} \mathrm{O}$. During anodic polarization in a $1 \mathrm{M} \mathrm{NaOH}$ solution, it was established [18] that a compact oxide film $\beta$ $\mathrm{Ni}(\mathrm{OH})_{2}$ is formed, which, after turning off the current, is slowly reduced to metallic nickel. In [19] the anodic passive films on nickel in $0.1 \mathrm{M} \mathrm{NaOH}$ have been studied by the methods of reflection and ellipsometry. With an increase in the potential, the thickness of the film increased from $1.1 \mathrm{~nm}$ to $2 \mathrm{~nm}$, and partial dehydration of the film and a change in its composition and structure took place. Thus, despite the huge number of works, there is no consensus regarding the processes of formation of oxide films on nickel, their structure and properties.

In this work, the behavior of nickel in a weakly alkaline medium is studied by the Electrochemical Impedance Spectroscopy (EIS) method. This method is more and more widely used to study corrosion processes [20-22]. It allows studying the structure of layers at the electrode-electrolyte interface for complex systems, as well as the mechanisms of reactions. This system was chosen as a model, since nickel is sufficiently stable in a weakly alkaline medium, which makes it possible to minimize the likelihood of a significant change in the properties of the electrode during a single measurement of the impedance spectrum. In addition, in an alkaline medium, almost all of the current during the anodic polarization of nickel is spent on the formation and thickening of the oxide-hydroxide layer. This makes it possible to exclude from consideration the reaction of the transition of nickel into solution. 


\section{Methods}

The object of study was a cylinder-shaped high-purity nickel sample with a geometric surface area of $3.62 \mathrm{~mm}^{2}$. Before the experiment, the sample was polished using M40 abrasive paper, degreased and fixed coaxially in a current lead holder isolated from the electrolyte. A solution of $0.001 \mathrm{M} \mathrm{NaOH}$ was prepared from "puriss." grade reagents and thermostated at $30^{\circ} \mathrm{C}$. The impedance measurements were carried out in a three-electrode electrochemical cell with natural aeration of the solution. Platinum served as an auxiliary electrode. The reference saturated silver chloride electrode contacts with the system through a bridge connection, excluding the penetration of $\mathrm{Cl}^{-}$ions into the working space of the cell. The following potentials are given relative to this electrode. IPC-Pro MF potentiostat with FRA-2 module was used for EIS studies. It provided the formation of constant and alternating current components of the polarization signal with the specified parameters and registration of the system response. The frequency dependences of the impedance were investigated in two modes:

- In the case of potentiostatic polarization, the base potential level was set, starting from the value of the open circuit potential of $-320 \mathrm{mV}$, then the electrode surface was subjected to cathodic reduction at $-640 \mathrm{mV}$ and $-420 \mathrm{mV}$, and then the potential was stepwise increased to $+600 \mathrm{mV}$, providing an anodic process of the oxide-hydroxide layer formation. During the polarization the frequency spectrum of the impedance was recorded at each potential level. The frequency range from $20 \mathrm{kHz}$ to $0.1 \mathrm{~Hz}$ with the modulating sinusoidal signal of $10 \mathrm{mV}$ was employed.

- In the case of galvanostatic polarization, some fixed levels of current densities were set sequentially on the sample: $0 \mu \mathrm{A} / \mathrm{cm}^{2}$ ("open circuit" mode), then in the cathodic direction: " $-1 "$, " $-2 ", "-5$ " and " $-7 " \mu \mathrm{A} / \mathrm{cm}^{2}$, and at last anodic currents: " $+1 ", "+2 "$, " $+5 "$ and " $+7 " \mu \mathrm{A} / \mathrm{cm}^{2}$. We used the $100 \mu \mathrm{A}$ current band at the IPC potentiostat in galvanostatic mode, ${ }^{1}$ and in this case, the amplitude of the sinusoidal signal was $\sim 0.1 \mu \mathrm{A} / \mathrm{cm}^{2}$. The impedance spectrums were recorded in the frequency range from $5 \mathrm{kHz}$ to $0.15 \mathrm{~Hz}$. The recording time for each frequency spectrum in this frequency range was about four minutes. At each current density level, two spectrums were obtained.

The EIS data obtained for each given potential or current density were processed by a specially developed DCS program ${ }^{2}$, which allows choosing the optimal structure of an equivalent circuit that adequately describes the experimental data, as well as calculating the values of its elements [23].

\footnotetext{
${ }^{1}$ In galvanostatic mode, the current range cannot be changed during polarization, in contrast to the potentiostatic mode where the current ranges can be switched at will, providing the best current sensitivity and frequency band of the complex.

${ }^{2}$ Dummy Circuits Solver (C 2020 developed by V.E. Kasatkin.
} 


\section{Results and Discussion}

Figure 1 compares the polarization curves obtained during EIS measurements of nickel samples in the alkaline medium. These curves represent the DC components of the signals obtained in EIS studies. Since the levels of DC signals varied somewhat depending on the current frequency and with time, two curves are presented for the conditions of potentiostatic polarization:

- the first curve for the initial moment of spectrum registration (at a frequency of $20 \mathrm{kHz}$ );

- the second curve, as averaging of DC current densities for all points of the spectrum (4 minutes at a given potential).

The character of the curves with a small current peak in the anodic region practically coincides with the anodic polarization curve for nickel at $\mathrm{pH} 11$ given in work [24].

Curve (3) corresponds to the galvanostatic conditions and the potentials correspond to the averaged DC levels during the recording of the impedance spectrum. The arrow shows the order of polarization application. The first point corresponds to the conditions of free corrosion (open circuit), then the electrode was cathodically polarized at $7 \mu \mathrm{A} / \mathrm{cm}^{2}$, after which it was switched to anodic polarization.

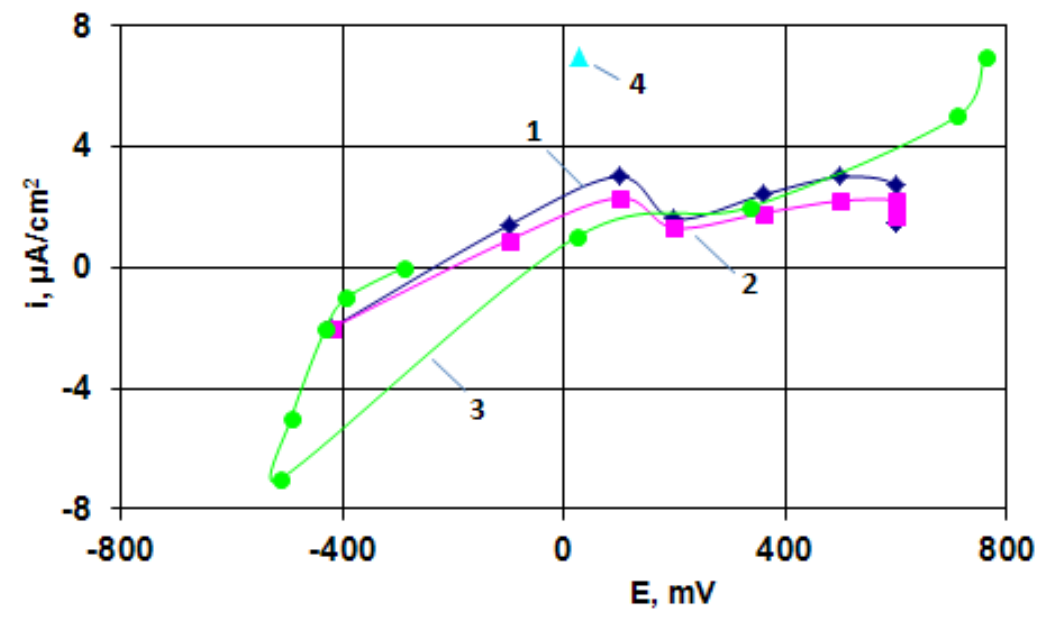

Figure 1. Potentiostatic $(1,2)$ and galvanostatic (3) polarization curves of nickel in $0.001 \mathrm{~N} \mathrm{NaOH}$. (4) - galvanostatic polarization after grinding of the electrode.

From Figure 1 it is evident, that the average current densities in potentiostatic mode in an alkaline medium, as a rule are somewhat lower than the initial currents. This is especially noticeable in the anodic region and is probably due to increased surface passivation during exposure at a given potential. But in general, the difference between the initial and average values of currents is not great in the potentiostatic mode. This allows us to assume that no significant changes in the structure of the electrode layers occur during the recording of the impedance spectrum. 
With the galvanostatic mode, higher current densities were provided both in the cathodic region (reducing conditions, although the potential for hydrogen evolution was not reached) and in the anodic one. However, the potentials at the beginning of the anodic region as a whole turned out to be comparable with the potentials at the similar of current densities under potentiostatic conditions without a characteristic peak on the anodic branch of the curve. At the anodic polarization above $5 \mu \mathrm{A} / \mathrm{cm}^{2}$, the electrode potential is 100 or more $\mathrm{mV}$ higher than the levels at the same current densities at the potentiostatic mode. The latter is due to the fact that, at the galvanostatic mode of polarization, the growth of the oxidehydroxide layer occurs in proportion to the applied current, and this causes an ohmic potential drop in the layer. If the film is removed mechanically by grinding on a sanding paper, then in the galvanostatic mode this leads to the expected decrease in polarization (at $+7 \mu \mathrm{A} / \mathrm{cm}^{2}$ by $732 \mathrm{mV}$ ) due to the destruction of the oxide and, accordingly, a decrease in the ohmic drop in the film (point 4).

The impedance spectra in potentiostatic experiments are shown in Figure 2 in Nyquist coordinates.

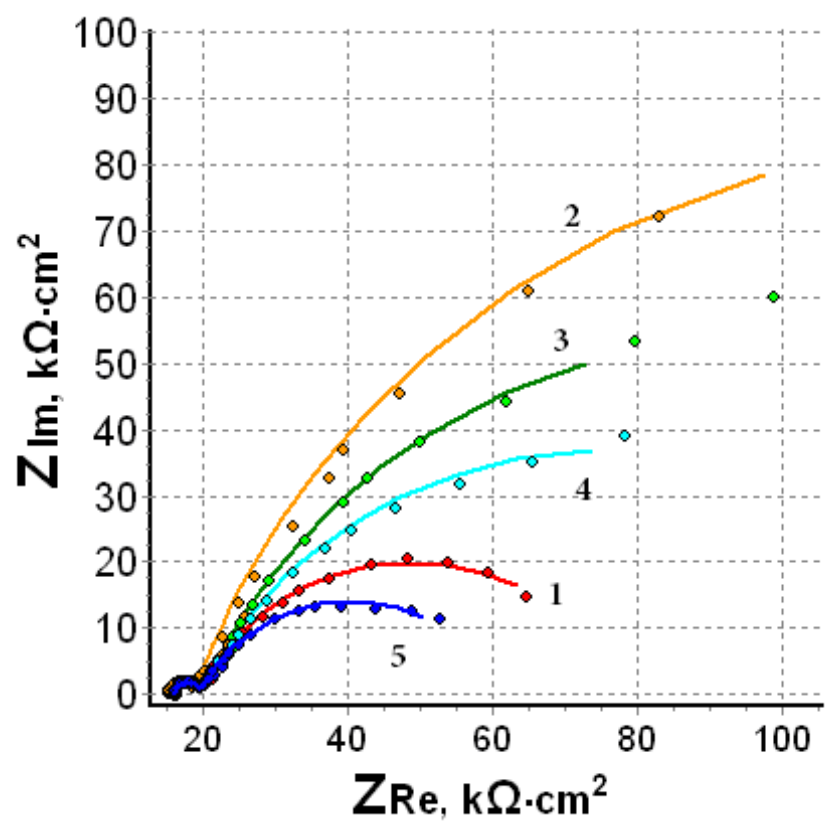

Figure 2. Nyquist plots in the frequency range $20 \mathrm{kHz}-0.1 \mathrm{~Hz}$. Ni in $0.001 \mathrm{M} \mathrm{NaOH}$. Potentiostatic mode: (1) $-E=-420 \mathrm{mV} ; I=-2.0 \mu \mathrm{A} / \mathrm{cm}^{2} ;(2)-E=-100 \mathrm{mV} ; I=+0.9 \mu \mathrm{A} / \mathrm{cm}^{2}$; (3) $-E=+200 \mathrm{mV} ; I=+1.3 \mu \mathrm{A} / \mathrm{cm}^{2} ;$ (4) $-E=+360 \mathrm{mV} ; I=+1.8 \mu \mathrm{A} / \mathrm{cm}^{2} ;(5)-E=+600 \mathrm{mV}$; $I=+2.2 \mu \mathrm{A} / \mathrm{cm}^{2}$. The dots mark the experimental data, the lines represent the results for the simulating equivalent circuit.

It can be seen that for all experiments in the potentiostatic mode that the shapes of the hodographs are similar, i.e., a small semicircle is observed in the high-frequency region from $20 \mathrm{kHz}$ to $100 \mathrm{~Hz}$ and a well distinguishable larger radius arc of semicircles appears in the low-frequency region. 


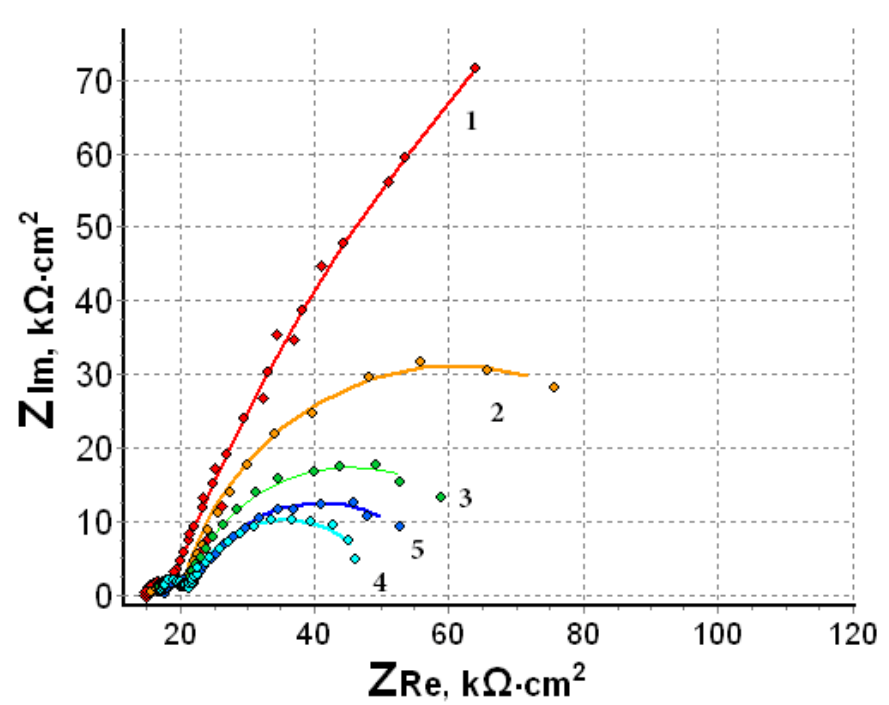

Figure 3a. Nyquist plots in the frequency range $5 \mathrm{kHz}-0.15 \mathrm{~Hz}$. Ni in $0.001 \mathrm{M} \mathrm{NaOH}$. Galvanostatic mode. Cathodic polarity: (1) $-E=-290 \mathrm{mV} ; I=0 \mu \mathrm{A} / \mathrm{cm}^{2}$ ("Open circuit"); (2) $-E=-393 \mathrm{mV} ; I=-1 \mu \mathrm{A} / \mathrm{cm}^{2}$; (3) $-E=-431 \mathrm{mV} ; I=-2 \mu \mathrm{A} / \mathrm{cm}^{2}$; (4) $-E=-493 \mathrm{mV} ; I=-$ $5 \mu \mathrm{A} / \mathrm{cm}^{2}$; (5) $-E=-513 \mathrm{mV} ; I=-7 \mu \mathrm{A} / \mathrm{cm}^{2}$. The dots mark the experimental data, the lines represent the results for the simulating equivalent circuit.

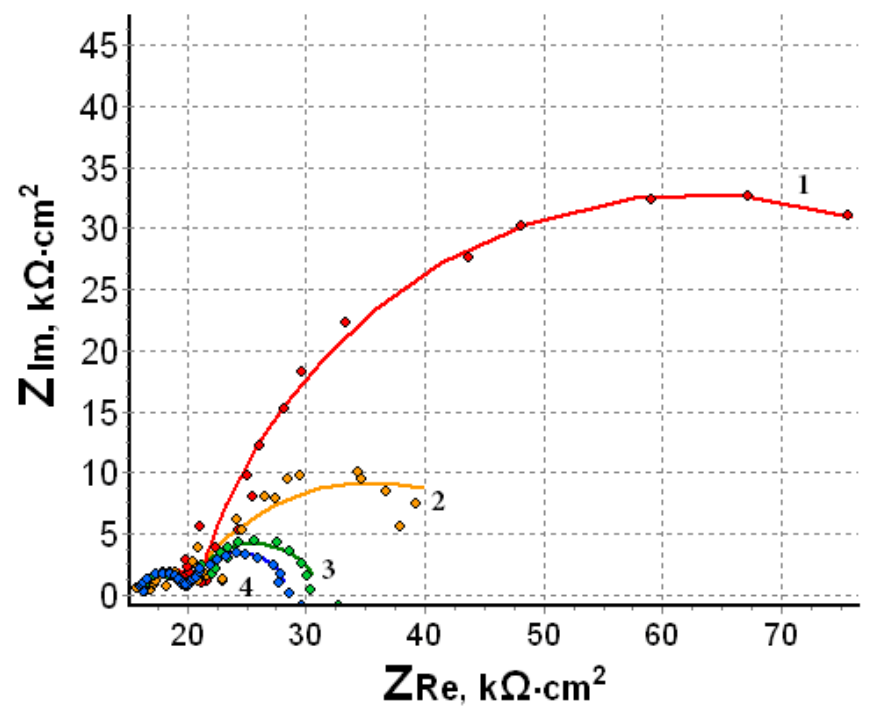

Figure 3b. Nyquist plots in the in the frequency range $5 \mathrm{kHz}-0.15 \mathrm{~Hz}$. Ni in $0.001 \mathrm{M} \mathrm{NaOH}$. Galvanostatic mode. Anodic polarity: (1) $-E=+22 \mathrm{mV} ; I=+1 \mu \mathrm{A} / \mathrm{cm}^{2}$; (2) $-E=+337 \mathrm{mV}$; $I=+2 \mu \mathrm{A} / \mathrm{cm}^{2}$; (3) $-E=+712 \mathrm{mV} ; I=+5 \mu \mathrm{A} / \mathrm{cm}^{2}$; (4) $-E=+762 \mathrm{mV} ; I=+7 \mu \mathrm{A} / \mathrm{cm}^{2}$. The dots mark the experimental data, the lines represent the results for the simulating equivalent circuit. 


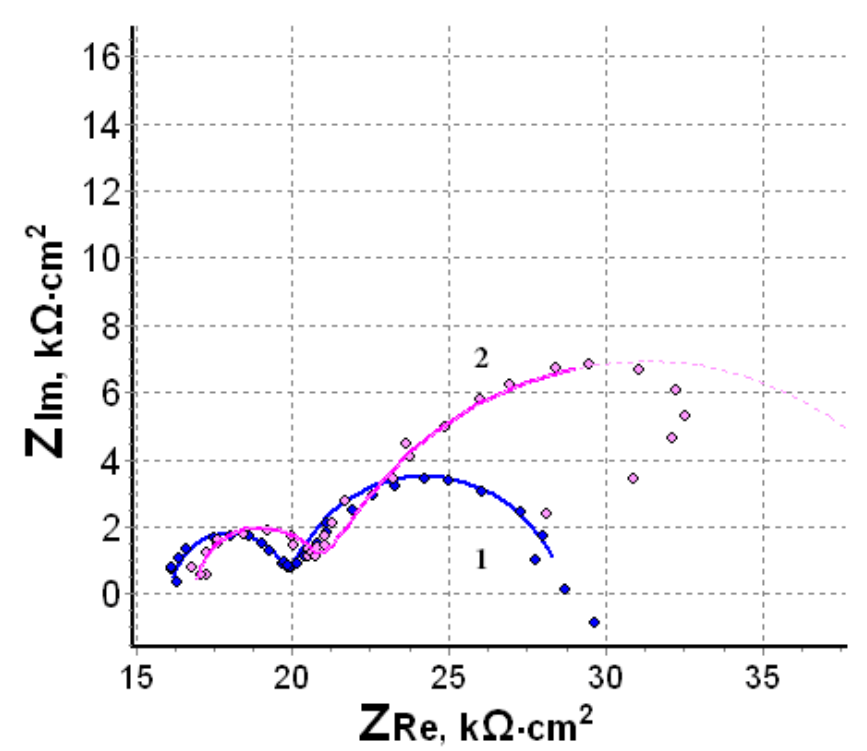

Figure 3c. Nyquist plots in the in the frequency range $5 \mathrm{kHz}-0.15 \mathrm{~Hz}$. Ni in $0.001 \mathrm{M} \mathrm{NaOH}$. Galvanostatic mode. Anodic polarity: (1) $-E=+762 \mathrm{mV} ; I=+7 \mu \mathrm{A} / \mathrm{cm}^{2}$; (2) $-E=+420 \mathrm{mV}$; $I=+7 \mu \mathrm{A} / \mathrm{cm}^{2}$ after grinding of the surface. The dots mark the experimental data. The solid lines show the results of simulation for the equivalent circuit in the range of $5 \mathrm{kHz}-1.4 \mathrm{~Hz}$. The dotted line shows the results of simulation for the full frequency range.

Figures $3 \mathrm{a}, 3 \mathrm{~b}$ and $3 \mathrm{c}$ represents similar frequency dependences of the impedance obtained in the galvanostatic mode. It can be seen that, both in the cathodic (Figure 3a) and in the anodic (Figure 3b, 3c) polarization regions, two semicircular arcs are also formed in the same frequency ranges as at the potentiostatic polarization. In contrast to the potentiostatic mode of EIS studies, a larger scatter of experimental points take place at the galvanostatic polarization, which is associated both with the nature of the films formation in this mode, and with the impossibility to change the current range at the IPC instrument (see note 1 above). In addition, when high anodic current densities are reached, the shape of the low-frequency semicircle becomes more and more distorted as the frequency decreases. This is clearly seen when the current density reaches $+7 \mu \mathrm{A} / \mathrm{cm}^{2}$ and especially on a freshly polished sample at the same anodic current density. In these experiments, the low-frequency semicircles were distorted, taking the form of a pseudo-inductive loop, which indicates an active dissolution of the metal (Figure 3c). A number of researchers believe that a progressive decrease in the real and imaginary components of the impedance vector with decreasing frequency (during the exposure time) is caused by discontinuity and a decrease in the protective properties of surface oxide layers, as a result of which pseudo inductive loops are formed in the low-frequency region, the appearance of which is explained by adsorption processes, and also by pitting corrosion [20, 25-28]. In our case, the appearance of the loop is more pronounced at relatively high current densities. If we bear in mind the study of the conductivity versus polarization [17], then it is logical to associate the pseudo inductive loop with an increase in the conductivity of the oxide-hydroxide layer. 


\section{The equivalent circuit of the process and the calculation of the nominal values of its elements}

Thus, for both potentiostatic and galvanostatic polarization, impedance frequency spectra require the models with at least two time constants. This makes it possible to apply the same type of equivalent circuits containing two parallel-connected RC groups of elements ${ }^{3}$ for modeling both polarization modes. At the same time, the use of a constant phase element (CPE) instead of a capacitor allows to describe better the systems with imperfect capacities caused by physicochemical and/or structural inhomogeneity of the charges distribution on their plates.

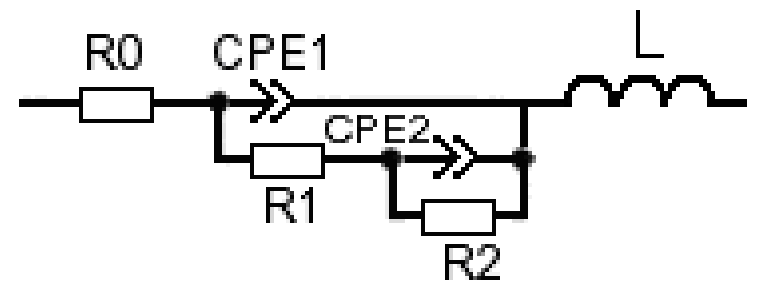

Figure 4. Equivalent circuit for simulation of EIS results.

In this study, we applied the circuit shown in Figure 4. In this circuit, $R 0$ corresponds to an ohmic potential drop in the electrolyte layer near the sample surface. $R 1$ and $R 2$ are the resistances of the high-frequency and low-frequency processes, respectively, and $C P E 1$ and $C P E 2$ are the elements of the constant phase for these processes. Thus, CPE1 element simulates the capacitive properties of the system at the outer boundary between the electrolyte and the oxide layers on the base metal (Ni). The CPE2 element corresponds to the capacity of the process associated directly with the electrochemical reaction occurring at the electrode surface, resulted from its polarization. The reaction rate is inversely proportional to the resistance $R 2$. Thus, $R 1$ in this model can take into account the volume inhomogeneity in the structure of the oxide a layer formed on the nickel surface and actually determines the ohmic potential drop in the oxide-hydroxide layer. Such an equivalent circuit structure is often used to simulate processes in systems with the surface layers, with a conductivity that is noticeably different from that for an electrolyte [20]. The inductive element $L$, which is formally present in the model, allows us to take into account the possible effects of inductive factors (wires, cell shape, instruments etc.) in the experiment, but in all our measurements it turned out to be negligible and was ignored in further calculations.

The results of modeling the frequency response on nickel electrode in an alkaline medium with potentiostatic and galvanostatic polarization are presented, respectively, in Tables 1 and 2. Although formally the modulus of the $C P E$ element has the dimension $\mathrm{Ohm}^{-1} \cdot \mathrm{c}^{\mathrm{n}} \cdot \mathrm{cm}^{-2}$, if the phase factor $n=1$, then it can be expressed in units of capacity $\mathrm{F} / \mathrm{cm}^{2}$. Based on the modeling results, it can be seen that the phase factor for the CPE1 element in

\footnotetext{
${ }^{3}$ These circuits can adequately describe only the frequency range without a pseudo-inductive arc appearance. The latter require a complication of the equivalent circuit and inclusion of additional elements in it.
} 
both cases is exactly equal to one, and approaches it for the CPE2 element. Therefore, for convenience of perception, we considered it possible to use capacity units for these elements, taking into account the above remarks.

Table $\mathbf{1}^{\mathbf{4}}$. Equivalent circuit elements values for potentiostatic measurement mode.

\begin{tabular}{|c|c|c|c|c|c|c|c|c|}
\hline$E, \mathbf{m V}$ & $\begin{array}{c}I, \\
\mu \mathrm{A} / \mathrm{cm}^{2}\end{array}$ & $\begin{array}{c}R 0, \\
\mathrm{k} \Omega \cdot \mathrm{cm}^{2}\end{array}$ & $\begin{array}{c}R 1, \\
\mathrm{k} \Omega \cdot \mathrm{cm}^{2}\end{array}$ & $\begin{array}{c}R 2, \\
\mathrm{k} \Omega \cdot \mathrm{cm}^{2}\end{array}$ & $\begin{array}{c}C P E 1 \\
Q_{0, \mu}, \mu \mathrm{F} / \mathbf{c m}^{2}\end{array}$ & $\underset{n}{C P E 1,}$ & $\begin{array}{c}C P E 2 \\
Q_{0}, \mu \mathrm{F} / \mathrm{cm}^{2}\end{array}$ & $\begin{array}{c}\text { CPE2, } \\
n\end{array}$ \\
\hline-320 & 0 & 14.92 & 3.57 & 671.53 & 0.076 & 1 & 10.83 & 0.75 \\
\hline-320 & -0.05 & 16.12 & 4.22 & 248.71 & 0.107 & 1 & 9.28 & 0.86 \\
\hline-320 & -2.10 & 15.90 & 4.34 & 217.75 & 0.102 & 1 & 8.71 & 0.87 \\
\hline-420 & -2.00 & 15.91 & 3.83 & 57.98 & 0.062 & 1 & 11.26 & 0.77 \\
\hline-100 & 0.90 & 15.35 & 4.40 & 215.51 & 0.089 & 1 & 9.24 & 0.83 \\
\hline 100 & 2.30 & 16.12 & 3.98 & 133.78 & 0.076 & 1 & 10.14 & 0.78 \\
\hline 200 & 1.30 & 16.11 & 3.99 & 161.18 & 0.082 & 1 & 10.22 & 0.78 \\
\hline 360 & 1.80 & 16,16 & 3.55 & 111.79 & 0.089 & 1 & 12.73 & 0.74 \\
\hline 500 & 2.20 & 15.93 & 3.59 & 61.47 & 0.085 & 1 & 15.70 & 0.73 \\
\hline 600 & 2.20 & 16.09 & 3.82 & 40.08 & 0.081 & 1 & 16.03 & 0.78 \\
\hline
\end{tabular}

Table 2. Equivalent circuit elements values for galvanostatic measurement mode.

\begin{tabular}{|c|c|c|c|c|c|c|c|c|}
\hline$E, \mathbf{m V}$ & $\begin{array}{c}I, \\
\mu \mathrm{A} / \mathbf{c m}^{2}\end{array}$ & $\begin{array}{c}R 0, \\
\mathbf{k} \Omega \cdot \mathbf{c m}^{2}\end{array}$ & $\begin{array}{c}R 1, \\
\mathrm{k} \Omega \cdot \mathrm{cm}^{2}\end{array}$ & $\begin{array}{c}R 2 \\
\mathrm{k} \Omega \cdot \mathrm{cm}^{2}\end{array}$ & $\begin{array}{c}C P E 1 \\
Q_{0,}, \mu \mathrm{F} / \mathrm{cm}^{2}\end{array}$ & $\begin{array}{c}C P E 1, \\
n\end{array}$ & $\begin{array}{c}C P E 2 \\
Q_{0}, \mu \mathrm{F} / \mathrm{cm}^{2}\end{array}$ & $\begin{array}{c}C P E 2, \\
n\end{array}$ \\
\hline-290 & 0 & 14.98 & 3.41 & 431.45 & 0.079 & 1 & 11.21 & 0.75 \\
\hline-393 & -1 & 16.36 & 3.85 & 80.44 & 0.064 & 1 & 9.12 & 0.84 \\
\hline-431 & -2 & 16.85 & 3.41 & 50.18 & 0.067 & 1 & 11.18 & 0.77 \\
\hline-493 & -5 & 17.31 & 3.91 & 29.06 & 0.065 & 1 & 12.27 & 0.78 \\
\hline-513 & -7 & 17.70 & 3.67 & 39.26 & 0.067 & 1 & 13.38 & 0.72 \\
\hline 22 & 1 & 16.67 & 4.18 & 84.42 & 0.076 & 1 & 11.34 & 0.84 \\
\hline 337 & 2 & 16.50 & 3.09 & 31.96 & 0.066 & 1 & 21.49 & 0.66 \\
\hline 712 & 5 & 16.32 & 3.49 & 11.70 & 0.073 & 1 & 16.47 & 0.79 \\
\hline 762 & 7 & 16.13 & 3.70 & 8.86 & 0.064 & 1 & $16.62^{5}$ & 0.85 \\
\hline $\begin{array}{c}\text { (Ggrinding) } \\
28\end{array}$ & 7 & 16.98 & 3.60 & 25.08 & 0.065 & 1 & 15.33 & 0.68 \\
\hline
\end{tabular}

\footnotetext{
${ }^{4}$ In Tables 1 and 2, the open circuit potential is highlighted in dark gray. The light gray background refers to the state after cathodic reduction at $-640 \mathrm{mV}$. The rest cells without background represent the anodic polarization.

${ }^{5}$ Frequency range from $5 \mathrm{kHz}$ to $1.4 \mathrm{~Hz}$.
} 
In all cases, the average relative discrepancy between the experimental and calculated values of the impedance vectors [23], as a rule, was within 1-2\% and only in some cases reached 5\% (with a strong scatter of experimental points or with a significant distortion of the semicircles shape). In some cases, as, for example, for a sample after grinding (Figure 3b), where the arc is strongly deformed in the region of the lowest frequencies, the frequency range for modeling was limited to the "problem-free region", i.e. frequency interval from $5 \mathrm{kHz}$ to $1.4 \mathrm{~Hz}$.

When comparing the results of Table 1 and Table 2, it can be seen that, in general, the values calculated for the equivalent circuit elements are very close for each parameter, regardless of the nature of the polarization mode "potentiostat" or "galvanostat". This confirms the correctness of the choice of the amplitude of the exciting harmonic signal that does not violate the quasilinear response of the system at the selected polarization levels [29].

The electrolyte resistance $R 0$ depends little on the polarization conditions and in both cases shows a tendency to a slight increase with an increase in both cathodic and anodic polarization. The value of the resistor $R 1$ is also insensitive to the polarization mode (potentiostat or galvanostat). Its value is proportional to the high-frequency arc diameter on Nyquist plot and slightly varies at a level $\sim 4 \mathrm{k} \Omega \cdot \mathrm{cm}^{2}$. From Figure 2 and Figure 3, it is evident that the initial high-frequency arcs are practically superimposed on each other in all polarization modes. It turned out that after grinding the surface, no noticeable effect on the resistor $R 1$. In any case, this resistor $R 1$ simulates some surface state that is not associated with Faraday's process, since it does not depend at all on the electrode polarization. It also cannot be unambiguously associated with the resistance of the oxide-hydroxide film, since there is no visible dependence on the film thickness associated with the potential and duration of polarization. It was assumed in the papers $[30,31]$ that this parameter is associated with the volume inhomogeneity of the oxide layer on the stainless steel 304 . This conclusion probably explains the absence of a clear dependence of the resistance $R 1$ on polarization for a two-layer film, when one of the layers remains constant in thickness. With a single-layer homogeneous film, this resistance is the sum of diffusion-migration ionic and electron-hole conductivities in the film, as well as charge transfer through the film-solution surface. In this case, its value is determined by the larger of the two series-connected resistances. Apparently, this determining resistance is the resistance to charge transfer across the film-solution interface, which is least associated with polarization.

Resistance $R 2$ turned out to be the most sensitive parameter to the applied polarization conditions. Its value varies within two orders of magnitude. The highest values refer to currentfree conditions (i.e. when the open circuit potential was maintained or the current was set to zero at the galvanostatic mode). In this case, $R 2$ turns out to be at the level of hundreds $\mathrm{k} \Omega$, and when both cathodic and anodic polarization are applied, it naturally decreases to tens or even several $\mathrm{k} \Omega$. This effect is especially pronounced during galvanostatic polarization, where higher current densities were used, and, therefore, the electrode reaction proceeded further from equilibrium and the reverse current was less affected. This feature of $R 2$ allows associating it with the polarization resistance of the electrochemical reaction of nickel 
oxidation (or reduction) at the applied external polarization. Indeed, if the reaction at the metal-film interface obeys the Tafel's equation, then:

$$
R 2=\delta E / \delta I=b / I
$$

where $E$ is the potential, $b$ is the coefficient in the Tafel's equation. In general, the dependence of the resistance on the reverse current is close to linear, but the values of " $b$ " are extremely high (about $1 \mathrm{~V}$ ). This indicates mixed control of the reaction. Apparently, there are diffusion restrictions in the film in the supply of anions and removal of cations.

The values of resistances $R 0, R 1$ and $R 2$ characterize the potential drop in these areas. Comparing their values, it should be noted that $R 2$ at low polarizations is an order of magnitude higher than $R 1$. In other words, the potential ohmic drop for the charge transfer across the metal-film interface is significantly higher than across the film-solution interface. But increasing the galvanostatic polarization cancels out this difference.

The capacitive properties of the system are determined by the phase-sensitive elements $C P E 1$ and $C P E 2$. Since the phase factor for both elements turned out to be close to unity, they can be considered as capacitors with varying degrees of "nonideality" of the plates. The $C P E 1$ element simulates the capacitance of a capacitor, which plates of are the metal surface of nickel and a layer of adsorbed ions in the electrolyte on the outer surface of the oxide layer. The $C P E 1$ module values were at the level of $0.06-0.10 \mu \mathrm{F} / \mathrm{cm}^{2}$. This is almost two orders of magnitude lower than the electrical double layer capacitance at the electrodes without surface films and makes it possible to evaluate in general the properties of the layers on the metal surface. It is interesting to note that with galvanostatic polarization in the "open circuit" mode and with cathodic polarity in the potentiostatic mode, the capacitance of these elements turns out to be somewhat higher, and in the anodic process, no significant differences are noticeable. Surface grinding has almost no effect on this parameter. It can also be assumed that, on a freshly grinded surface, the thickness of the air oxide layer and the layer after anodic polarization are of the same order at the studied process durations. However, there are differences in the internal structure, which is noticeable when comparing the values of the $C P E 2$ element. In conclusion, it should be noted that, since the phase factor of the $C P E 1$ element is almost exactly equal to unity, this element can be considered a perfect capacitor. Such element is described well enough within the framework of the model with two boundaries and, accordingly, with three capacitors connected in series. These are capacities at the interface between the metal and the oxide-hydroxide film, the interface between this layer and the solution, as well as the capacitance formed by the hydroxide film itself with two plates from the two above-mentioned capacitors. The capacitance measured by the electrochemical impedance method is actually close to the smallest of the three listed. The first capacitance is large and does not affect the measurement at high frequencies. The capacitance of the film with two plates should depend on the film thickness and change with polarization, which is not noted. Consequently, the measured capacitance corresponds to the film-solution interface and is an almost ideal small capacitor responsible for the high- 
frequency semicircle in the Nyquist diagram. Unfortunately, the capacity of the coated film is not determined in in situ experiments of this kind.

The CPE2 element in the discussed model refers to the capacitive process at the interface between the metal and the inner regions of the oxide layer where the Faraday process of nickel oxidation or reduction takes place, depending on the direction of the external current. It belongs to the area where the main potential jump is localized, and according to the $C P E 2$ modulus level, which mainly are within the range of $10-20 \mu \mathrm{F} / \mathrm{cm}^{2}$, it can be assumed as a capacitor with very close plates. This component causes the appearance of a large semicircle at the low frequencies. There is a definite dependence on the polarization conditions, although not entirely unambiguous. In general, it can be seen that there is some correlation between the increase in current density and the level of the $C P E 2$ module, especially at the galvanostatic mode of polarization. The phase factor "CPE2 $n$ " in the potential ranges until the breakdown of the film is achieved and the equivalent circuit is adequate to the experiment basically falls within the range of $0.7-0.9$, which can be due to some inhomogeneity in the structure of the capacitor. At the higher anodic potentials the film breakdown was happened, so the discussed model ceases to be adequate to experiment and the formally calculated values of the phase factor are not correct.

\section{Conclusion}

Thus, by EIS method, the formation of a surface layer on nickel surface in a weak alkaline medium is established, the electrical characteristics of which are practically not affected by the polarization conditions in the investigated potential range, cathodic and anodic current densities. An equivalent circuit has been chosen that adequately describes the behavior of nickel electrode in the frequency range $20 \mathrm{kHz}-0.1 \mathrm{~Hz}$. The nominal values of the elements of this circuit were calculated for various polarization conditions. The resistivity of this surface layer has been determined, which can be associated with oxide-hydroxide layers formed on nickel and discussed in the papers. Also, the parameters of the circuit elements corresponding to the polarization resistance and the capacity of redox reactions at the electrode were estimated. These studies show that using EIS measurements it is quite well possible to distinguish two processes that contain a capacitive component: the Faraday process of nickel oxidation reaction at the metal-oxide interface and the process of ion charge transfer across the oxide-solution interface. According to the calculated resistance values, in principle, it is possible to estimate the true value of the potentials in the electrochemical reaction zone. These studies will require additional refinement experiments that are planned in the future.

\section{References}

1. K.A. Chandler et al., Corrosion: Handbook, Moscow, Metallurgiya, 1981, 632 (in Russian).

2. F. Todt, Corrosion and corrosion protection, Leningrad, Khimiya, 1966, 848 (in Russian). 
3. Tr. Kurgan Engineering Institute, Electrochemistry and corrosion, vol. 17, Kurgan, 1971, 103 (in Russian).

4. M.A. Dasoyan, Chemical Power Sources: A Reference Book, $2^{\text {nd }}$ ed., Leningrad, Energiya, 1969, 587 (in Russian).

5. V.S. Bagotsky and A.M. Skundin, Chemical current sources, Moscow, Energoizdat, 1981, 360 (in Russian).

6. I.A. Sizova, S.I. Serdyukov and A.L. Maksimov, Nickel-tungsten sulfide catalysts obtained in situ in a hydrocarbon medium for hydrogenating aromatic hydrocarbons, Neftekhimiya, 2015, 55, no. 4, 319-331 (in Russian).

7. Z.A. Sabirova, M.M. Danilova, V.I. Zaikovsky, N.A. Kuzin et al., Nickel catalysts based on porous nickel for the steam conversion of methane, Kinet. Katal., 2008, 49, no. 3, 449-456 (in Russian). doi: 10.7868/S0028242115040115

8. N.D. Tomashov and G.P. Chernova, Corrosion theory and corrosion resistant structural alloy, Moscow, Metallurgiya, 1986, 359 (in Russian).

9. E. Sussek and M. Kesten, Eine Charakterisierung der Lochfraßkorrosion des Nickels I. Die Bestimmung der Lochfraßpotentiale in neutralen und alkalischen Lösungen, Corros. Sci., 1975, 15, no. 4, 225-238. doi: 10.1016/S0010-938X(75)80018-7

10. A.G. Pshenichnikov, Z.I. Kudryavtseva, L.A. Burkaltseva et al., Investigation of the state of the surface of a nickel electrode by ellipsometric and potentiodynamic methods, Elektrokhimiya, 1980, 16, no. 2, 161-165 (in Russian).

11. Z.I. Kudryavtseva, I.A. Openkin, N.A. Zhuchkova, E.I. Khrushcheva and N.A. Shumilova, Study of oxygen adsorption on nickel in alkaline electrolytes by ellipsometric methods, Elektrokhimiya, 1975, 11, no. 10, 1488-1492 (in Russian).

12. N. Sato and K. Kudo, An ellipsometric study of anodic passivation of nickel in borate buffer solution, Electrochim. Acta, 1974, 19, no. 8, 461-470. doi: 10.1016/00134686(74)87025-8

13. S. Shah, Surface and interface characterization in corrosion, Houston, NACE International, 1994, 179.

14. H.-H. Strechblow, Nucleation and repassivation of corrosion pits for pitting on iron and nickel, Mater. Corros., 1976, 27, no. 11, 792-799.

15. N. Sridhar and D.S. Dunn, In situ study of salt film stability in simulated pits of nickel by raman and electrochemical impedance spectroscopies, J. Electrochem. Soc., 1997, 144, no. 12, 4243-4253. doi: $\underline{10.1149 / 1.1838173}$

16. Z.I. Kudryavtseva, L.A. Burkaltseva and A.G. Pshenichnikov, Application of the ellipsometric method for studying the surface properties of nickel electrodes in alkaline electrolyte, Elektrokhimiya, 2004, 40, no. 11, 1409-1415 (in Russian).

17. W.Visscher and E. Barendrecht, Anodic oxide films of nickel in alkaline electrolyte, Surface Sci., 1983, 135, no. 1-3, 436-452.

18. L.M.M. de Souza, F.P. Kong, F.R. McLarnon and R.H. Muller, Spectroscopic ellipsometry study of nickel oxidation in alkaline solution, Electrochim. Acta, 1997, 42, no. $8,1253-1267$. 
19. Woon-kie Paik, Z. Szklarska-Smialowska, Reflectance and ellipsometric study of anodic passive films formed on nickel in sodium hydroxide solution, Surface Sci., 1983, 96, 401-412.

20. D.D. Macdonald, Some advantages and pitfalls of electrochemical impedance spectroscopy, Corros. Sci., 1990, 46, no. 3, 229-242.

21. S.S. Vinogradova, I.O. Iskhakova, R.A. Kaidrikov and B.L. Zhuravlev, Method of impedance spectroscopy in corrosion research, Publishing house of KNITU, Kazan, 2012, 96 (in Russian).

22. J.-P. Diard, C. Montella and N. Murer, Handbook of electrochemical impedance spectroscopy. Corosion reactions library, LEPMI, Bio-Logic, 2012, 23.

23. A.I. Shcherbakov, I.G. Korosteleva, I.V. Kasatkina, V.E. Kasatkin, L.P. Kornienko, V.N. Dorofeeva, V.V. Vysotskii and V.A. Kotenev, Impedance of aluminum electrode with nanoporous oxide, Prot. Met. Phys. Chem. Surf., 2019, 55, no. 4, 689-694. doi: 10.1134/S2070205119040208

24. R.K. Astakhova, A.A. Belustin, V.V. Berenblit et al., Handbook of electrochemistry, Khimiya, 1981, 488 (in Russian).

25. N. Mahato and M.M. Singh, Investigation of passive film properties and pittinp resistance of ALSI 316 in aqueous ethanoic acid containing chloride ions using elextrochemical impedance spectroscopy (EIS), Port. Electrochim. Acta, 2011, 29, no. 4, 233-251. doi: 10.4152/pea.201104233

26. R. Ittah, E. Amsellem and D. Itzhak, Pitting corrosion evaluation of titanium in $\mathrm{NH}_{4} \mathrm{Br}$ solutioms by electrochemical methods, Int. J. Electrochem. Sci., 2014, 9, no. 2, 633643.

27. M. Itagaki, A. Taya, K. Watanabe and K. Noda, Deviations of capacitive and inductive loops in the electrochemical impedance of a dissolving iron electrode, Anal. Sci., 2002, 18, no. 6, 641-644. doi: 10.2116/analsci.18.641

28. I. Annergren, D. Thierry, F. Ziu, Localized electrochemical impedance spectroscopy fpr studying pipping corrosion on stainless steels, J. Electrochem. Soc., 1997, 144, no. 4, 1208-1214. doi: $10.1149 / 1.1837574$

29. Z.B. Stoinov, B.M. Grafov et al., Electrochemical impedance, Moscow, Nauka, 1991, 3-35 (in Russian).

30. S. Krakowiak, K. Darowicki and P. Ślepski, Evaluation of pitting corrosion by means of dynamic electrochemical impedance spectroscopy, Electrochim. Acta, 2004, 49, no. 1718, 2909-2918. doi: 10.1016/j.electacta.2004.01.070

31. S. Krakowiak, K. Darowicki and P. Ślepski, Impedance of metastable pitting corrosion, J. Electroanal. Chem., 2005, 575, no. 1, 33-38. doi: 10.1016/j.jelechem.2004.09.001 